\title{
«Un esame, due diplomi»: l'ESABAC, programma binazionale italo-francese
}

Risultati di una ricerca empirica nella regione Toscana

\section{Valentina Rocchi e Gérald Schlemminger}

\section{(2) OpenEdition}

\section{Edizione digitale}

URL: http://journals.openedition.org/esp/538

DOI: $10.4000 /$ esp.538

ISSN: 2532-0319

\section{Editore}

Centre d'Information sur l'Éducation Bilingue et Plurilingue

Edizione cartacea

Data di pubblicazione: 1 juin 2015

Paginazione: 47-58

ISSN: 1127-266X

Notizia bibliografica digitale

Valentina Rocchi e Gérald Schlemminger, ««Un esame, due diplomi»: I’ESABAC, programma

binazionale italo-francese», Éducation et sociétés plurilingues [Online], 38 | 2015, Messo online il 01 mars 2016, consultato il 25 février 2021. URL: http://journals.openedition.org/esp/538 ; DOI: https:// doi.org/10.4000/esp.538 


\section{«UN ESAME, DUE DIPLOMI»: L'ESABAC, PROGRAMMA BINAZIONALE ITALO-FRANCESE. RISULTATI DI UNA RICERCA EMPIRICA NELLA REGIONE TOSCANA}

\section{Valentina RocGHi, Gérald Schlemminger}

Le travail met en relief les atouts et les faiblesses de l'Esabac, programme d'éducation binationale italo-français, en Toscane. Cette région italienne présente en fait une hétérogénéité par rapport aux établissements scolaires ayant activé ce projet. Dans la partie introductive, le dispositif est introduit à partir des dispositions législatives, qui ont mené à sa création et qui l'ont promu au niveau national. Par la suite, la contribution se plonge dans son étude empirique, constituée par une enquête proposée aux enseignants et aux élèves de classes de terminale de six établissements scolaires toscans. L'analyse se divise ainsi en une étude qualitative impliquant 19 enseignants et en une étude quantitative impliquant 178 élèves.

Diese Arbeit möchte ein Gesamtbild der positiven und negativen Aspekte des Esabacs, binationales italienischfranzösisches Abitur, in der Toskana geben. Diese italienische Region wurde aufgrund der Heterogenität, was die Auswahl an dem Programm teilnehmenden Schulen angeht, ausgesucht. Im ersten Teil wird der Esabac im Zusammenhang zu den Rechtsvorschriften, die zu seiner Gründung und Einführung im italienischen Schulsystem gesorgt haben, vorgestellt. Desweiteren widmet sich dieser Beitrag einer empirischen Studie, die aus zwei Befragungen besteht, eine für die 19 Professorinnen und Professoren der sechs ausgewählten Schulen und eine für die 178 Schülerinnen und Schüler. Die entsprechenden Analysen werden jeweils durch einen qualitativen und einen quantitativen Forschungsansatzes durchgeführt.

The strong and weak points of the binational program in Italian and French, the Esabac, appear in research carried out in Tuscany, an Italian region marked by much diversity in the schools that actively participated in the project. Our introduction first presents the system based on the legislation that presided at the creation of the Esabac and promoted it to a national level. The empirical part of our research is a survey done among the teachers and last-year pupils of six Tuscan schools. Our analysis thus consists in a qualitative study with 19 teachers and a quantitative study involving 178 students.

Key-words: ESABAC, Italian-French diploma, qualitative study, Tuscany, statistics

INTRODUZIONE ALL'ESABAC

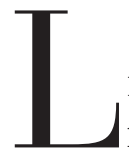

'Esabac è un doppio diploma, con il quale gli alunni del triennio del ciclo di istruzione superiore di primo grado francese e italiano ottengono il rilascio contemporaneo del diploma di maturità e del Baccalauréat. Gli alunni partecipanti al dispositivo hanno così la possibilità di accedere al ciclo di istruzione superiore di secondo grado di entrambi i paesi. Questo percorso di studio è per la 
«Un esame, due diplomi»: I'ESABAC, una ricerca empirica nella regione Toscana

V. ROCCHI, G. SCHLEMMINGER prima volta nominato nell'accordo intergovernativo italo-francese del 2009, in seguito approfondito e integrato, per la parte italiana, dai decreti ministeriali (DM) 91/2010 e 95/2013, e, per la parte francese, nel Bulletin Officiel spécial n 5 del 17 giugno 2010 (arrêté 2.6.2010). Tra i vari obiettivi dell'Esabac, i principali sono: "la promozione della cooperazione culturale, in particolare nel campo educativo, con iniziative mirate a stabilire legami tra i sistemi educativi dei due Stati; il rafforzamento dell' insegnamento della lingua e della cultura dell'altro Paese; l'apportare di un contributo importante alla cooperazione e all'integrazione europee (accordo interg. 2009: 1)".

La durata del percorso copre gli ultimi tre anni degli istituti superiori di primo grado italiani e francesi. Le materie specifiche, da trattare in italiano nelle scuole francesi ed in francese negli istituti italiani, sono la lingua e letteratura ed una disciplina non linguistica, ovvero la storia nel caso dell'Italia (insegnata per 2 ore settimanali) e la storia/geografia nel caso della Francia (insegnata per 3 ore settimanali in terza e per 4 ore nelle classi quarta e quinta). Alla fine del percorso, gli studenti raggiungono il livello B2 in lingua straniera del quadro comune europeo di riferimento per le lingue (QCER).

Gli istituti che possono aderire al dispositivo sono, nel caso della Francia, le sezioni binazionali, facenti parti delle voies générales L (letteratura), ES (economica-sociale) e S (scientifica). Nel caso dell'Italia invece, gli istituti presi in considerazione sono quelli riformati nel 2010 dall'allora ministro dell'istruzione Gelmini. Quindi, nel caso degli istituti tecnici, quelli corrispondenti appartengono al settore economico, indirizzo amministrazione, finanza e marketing oppure turismo. Per quel che riguarda i licei invece, l’Esabac può concernere i linguistici, classici, artistici, scientifici, musicali e coreutici e delle scienze umane.

La strutturazione dell'esame finale è descritta, per la parte italiana negli allegati nr. 5 dei DM 91 e 95 e, per la parte francese, nel Bulletin Officiel n 46 del 16 dicembre 2010 - note de service n 2010-227 du 30-112010. Nel caso della lingua e letteratura, le prove da superare sono due: la prima, scritta, è di 4 ore sia in Francia che in Italia (possibilità di scegliere tra un'analisi testuale o un saggio breve a partire dallo studio di un insieme di documenti), la seconda, orale, prevede una durata di 40 minuti in Francia, ed è, invece, inserita nel colloquio dell'Esame di Stato, in Italia. Per quel che riguarda la storia invece, al momento della prova scritta, gli alunni devono scegliere tra una composizione e lo studio e l'analisi di un insieme di documenti. In questo caso la durata è di 5 ore per la Francia e di 2 ore per l'Italia.

$\mathrm{Al}$ momento di questo studio, ovvero durante l'anno scolastico (a.s.) 2013/2014, le sezioni Esabac sono presenti in 244 scuole in Italia e 
«Un esame, due diplomi»: I'ESABAC, una ricerca empirica nella regione Toscana

V. ROCch, G. SCHLEMMINGER

CONTESTO

E OBIETTIVI

DELLA RICERCA

PRESENTAZIONE DEI RISULTATI DEL QUESTIONARIO AGLI INSEGNANTI
42 in Francia. Nel contesto di questa indagine, l'analisi del dispositivo è circoscritta ai 24 istituti della regione Toscana.

L'obiettivo principale del testo qui presentato è quello di ottenere un quadro generale delle forze e delle debolezze dell'Esabac in Toscana. In questo contesto sono stati presi in considerazione due sondaggi, entrambi svolti durante l'a.s. 2013/2014: il primo rivolto agli insegnanti ed il secondo agli alunni. Nel primo caso, le domande poste riguardano l'esperienza professionale nonché l'approccio metodologico e linguistico utilizzato in classe. Vengono inoltre richieste informazioni riguardo l'organizzazione delle lezioni così come una valutazione personale del dispositivo. Nel caso, invece, degli alunni, l'obiettivo posto è quello di ottenere un quadro delle motivazioni che li hanno spinti a scegliere il percorso binazionale, ma anche di conoscere più a fondo le loro ambizioni dopo l'ottenimento del doppio diploma. Un altro aspetto messo in evidenza è inoltre l'offerta di attività curriculari ed extracurriculari in rapporto con la lingua francese nonché il perseguimento personale dell'approfondimento della lingua straniera $(1)$.

Nel caso degli insegnanti, il numero totale è di 19. I questionari agli alunni sono invece stati consegnanti a 178 persone, la maggior parte dei quali (110) è iscritta al liceo "Machiavelli-Capponi" a Firenze (questo istituto presenta 7 classi quinte con sezione Esabac). Dati sul rapporto tra maschi e femmine, nonché tra bilingue e monolingue riguardanti gli alunni di tutti gli istituti sono:

- $13 \%$ maschi, $87 \%$ femmine;

- $10 \%$ femmine bilingui, $77 \%$ femmine monolingui, $2 \%$ maschi bilingui, $11 \%$ maschi monolingui.

I due sondaggi sono stati svolti seguendo, per i docenti, un approccio qualitativo, per gli alunni invece, un approccio quantitativo. In entrambi i casi, i questionari sono stati fatti riempire anonimamente. Per garantire una visione più oggettiva possibile dell'attuazione dell'Esabac, una persona di riferimento è stata sempre presente durante la trasmissione alle classi.

Adesso che contesto e obiettivo della ricerca sono stati messi in evidenza, il prossimo capitolo si occuperà di riportare i risultati principali del sondaggio agli insegnanti.

Come introdotto nel capitolo precedente, l'inchiesta rivolta al corpo docente prende in considerazione un totale di 19 candidati (4 uomini e 15 donne): 10 (2 uomini e 8 donne) si occupano della lingua e della letteratura francese; i restanti 9 (2 uomini e 7 donne) insegnano invece storia. Prima di descrivere i risultati principali è utile presentare in 
«Un esame, due diplomi»: I'ESABAC, una ricerca empirica nella regione Toscana

V. ROCCH, G. SCHLEMMINGER

questa sede alcune informazioni generali riguardante il profilo dei candidati:

- La maggioranza degli insegnanti di lingua e letteratura (6/10) insegna da meno di 5 anni. I colleghi di storia praticano invece la professione da un periodo compreso tra i 10 ed i 20 anni.

- Dal 2010 il MIUR (Ministero dell'Istruzione dell'Uniwersità e della Ricerca) propone, ai docenti di discipline non linguistiche, la possibilità di partecipare a dei corsi di formazione CLIL (Content and Language Integrated Learning). Nel contesto di questa ricerca, soltanto una persona si avvale di questa offerta.

- Per quel che riguarda le competenze in lingua francese dei docenti di storia, 2 sono madrelingua, 2 presentano un livello B2, 1 un livello B1 ed 1 non dispone di alcun diploma di lingua francese.

Nel contesto dei risultati da presentare, uno dei primi aspetti trattati nel questionario prende in considerazione se gli insegnanti riescano a trasmettere più o meno correttamente gli aspetti principali del programma Esabac. La domanda è stata dunque strutturata avvalendosi di una scala "Likert", la quale spazia tra molto facile a molto difficile. La tabella seguente mostra i risultati principali nel caso degli insegnanti di lingua e letteratura francese:

MOLTO FACILE

Utilizzazione costante della lingua francese in classe

Studio approfondito della letteratura francese

Utilizzazione corretta metodologia per la creazione dei testi.

Come è possibile constatare nello schema sovrastante, le difficoltà maggiori riguardano il trattamento della parte del programma in rapporto sia con l'Italia che con l'Europa ed il mondo.

Nel caso dei colleghi di storia invece, la tendenza delle risposte date è piuttosto positiva: la maggioranza corrisponde infatti ad abbastanza facile, in particolare la valutazione degli alunni.

Di seguito, il questionario si concentra sull'integrazione della metodologia Esabac nei corsi delle discipline specifiche. Come risultato, la maggioranza degli insegnanti giudica di riuscire a trasmettere correttamente questi principi. L'unico punto critico corrisponde al completo trattamento del programma durante l'a.s. 
«Un esame, due diplomi»: I'ESABAC, una ricerca empirica nella regione Toscana

V. ROCCH, G. SCHLEMminger
Un'ulteriore domanda si concentra sulla frequenza di lezioni interdisciplinari in classe. A riguardo, i risultati mettono in evidenza una netta differenza tra gli insegnanti di lingua e letteratura e di storia. Nel primo caso vi è una maggiore pratica di attività interdisciplinari: da un massimo di una volta a settimana (3/10) ad un minimo di ogni due mesi (1/10). La maggioranza dei colleghi di storia (7 su 9) lavora invece in maniera interdisciplinare meno di una volta al mese. Le restanti due persone confermano questa tendenza negativa, in quanto una di loro ha dato come risposta ogni due mesi, l'altra invece mai.

Risultati significativi sono stati inoltre, ottenuti da un'ulteriore domanda riguardante la frequenza di contatti tra gli insegnanti ed i colleghi di altri istituti Esabac in Italia. La tendenza è negativa: le risposte si situano infatti tra meno di una volta al mese e mai. È stato inoltre chiesto se i docenti scambiassero tra di loro del materiale didattico. A riguardo i risultati indicano che sia nel caso dei candidati di lingua e letteratura che nel caso dei colleghi di storia, solamente la metà (5 persone per ognuno dei due gruppi) intrattengono questi scambi.

Per il momento i libri di testo riguardanti l'Esabac sono stati promossi senza responsabilità dell'ambasciata e del MIUR. Inoltre, per quel che riguarda la storia, non esiste un manuale ufficiale per il dispositivo. In linea con ciò, nel questionario è stata inserita una domanda riguardante i materiali didattici, in termini di manuali ed in termini di integrazione ad essi, di cui si servono gli insegnanti (2). Sono di seguito messi in evidenza i risultati riguardanti i motivi per i quali gli insegnanti hanno deciso di lavorare nelle sezioni Esabac. Come d'abitudine, la domanda è stata rivolta separatamente ai docenti di lingua e letteratura e a quelli di storia. Di seguito la tabella indicante

\begin{tabular}{|l|c|c|c|c|c|c|c|}
\hline & $\begin{array}{c}\text { Assoluta } \\
\text { mente s̀̀ }\end{array}$ & Sì & $\begin{array}{c}\text { Abbas } \\
\text { tanza }\end{array}$ & $\begin{array}{c}\text { Indeci } \\
\text { so/a }\end{array}$ & $\begin{array}{c}\text { Non } \\
\text { abbast } \\
\text { anza }\end{array}$ & No & $\begin{array}{c}\text { Assoluta } \\
\text { mente no }\end{array}$ \\
\hline Interesse per la lingua e la cultura francese & 8 & 2 & & & & & \\
\hline $\begin{array}{l}\text { Possibilità di insegnare un altra materia (storia, } \\
\text { letteratura) in lingua francesc. }\end{array}$ & 5 & 3 & & & & & 2 \\
\hline $\begin{array}{l}\text { Possibilità di continuare ad insegnare nello } \\
\text { stesso istituto. }\end{array}$ & 4 & 2 & & & 2 & & 2 \\
\hline $\begin{array}{l}\text { Mi è stato chiesto di insegnare nella sezione } \\
\text { Esabac. }\end{array}$ & 4 & 1 & & & 1 & 1 & 3 \\
\hline Nessuna particolare motivazione. & & & & & 1 & 1 & 6 \\
\hline
\end{tabular}

le risposte del primo gruppo:

Come è possibile dedurre dalla tabella, 4 persone hanno indicato di 
insegnare nell'Esabac 1) di modo da continuare a lavorare nello stesso istituto scolastico e 2) perché è stato loro domandato da detto istituto. Nel caso degli insegnanti di storia, questa seconda opzione è stata indicata dalla maggioranza. Ulteriori risultati riguardanti questo

\begin{tabular}{|l|c|c|c|c|c|c|c|}
\hline & $\begin{array}{c}\text { Assoluta } \\
\text { mente sì }\end{array}$ & Sì & $\begin{array}{c}\text { Abbas } \\
\text { tanza }\end{array}$ & $\begin{array}{c}\text { Indeci } \\
\text { so/a }\end{array}$ & $\begin{array}{c}\text { Non } \\
\text { abbast } \\
\text { anza }\end{array}$ & No & $\begin{array}{c}\text { Assoluta } \\
\text { mente no }\end{array}$ \\
\hline Intercsse per la lingua c la cultura francesc & 3 & $\mathbf{5}$ & 1 & & & & \\
\hline $\begin{array}{l}\text { Possibilità di insegnare un'altra materia (storia, } \\
\text { letteratura) in lingua francese. }\end{array}$ & 2 & $\mathbf{6}$ & & & 1 & & \\
\hline $\begin{array}{l}\text { Possibilità di continuare ad insegnare nello } \\
\text { stesso istituto. }\end{array}$ & 1 & $\mathbf{2}$ & 1 & 1 & 1 & $\mathbf{2}$ & 1 \\
\hline $\begin{array}{l}\text { Mi è stato chiesto di insegnare nella sezione } \\
\text { Esabac. }\end{array}$ & $\mathbf{5}$ & 1 & & & 1 & & 2 \\
\hline Nessuna particolare motivazione. & & 1 & & & 1 & & 6 \\
\hline
\end{tabular}

«Un esame, due diplomi»: I'ESABAC, una ricerca empirica nella regione Toscana

V. ROCCHI, G. SCHLEMMINGER

PRESENTAZIONE DEI RISULTATI DEL QUESTIONARIO AGLI ALUNNI gruppo sono osservabili nella tabella seguente:

Ai candidati è stato infine richiesto un apprezzamento ed una valutazione dell'Esabac. Le risposte a riguardo indicano una tendenza positiva: la maggioranza ( 6 su 10 nel caso della lingua e letteratura e 7 su 9 per la storia) è abbastanza soddisfatta. Tra gli eventuali miglioramenti da esercitare vi è la necessità di un approfondimento degli aspetti linguistici e metodologici tramite una maggiore formazione del personale; è inoltre indicata una riduzione del programma e, contemporaneamente, un approfondimento di quelle parti che trattano della storia e della letteratura italiana. Infine, gli insegnanti desidererebbero ottenere dal MIUR uno statuto di docenti-Esabac.

In questo contesto è stato proposto un riassunto sui risultati principali del questionari agli insegnanti. Il prossimo capitolo si concentrerà quindi sulla presentazione dell' inchiesta agli alunni delle classi quinte.

La seconda parte di questa analisi riguarda un sondaggio a 178 alunni delle classi quinte di 6 istituti scolastici toscani riportanti delle sezioni Esabac. Per poterne riassumere al meglio i risultati principali, è stata operata una selezione degli aspetti da trattare, motivo per il quale non è stato possibile considerare il questionario nella sua totalità, sia per quel che riguarda i singoli quesiti sottoposti agli allievi, sia in riferimento all'analisi statistica dei risultati (per ogni domanda posta è stata applicata un'analisi della significatività, di modo da poter osservare se le risposte ottenute possono riguardare un pubblico più vasto oppure se sono dovute al caso). 
«Un esame, due diplomi»: I'ESABAC, una ricerca empirica nella regione Toscana

V. Rocchi, G. SCHLEMmingeR
Uno dei primi aspetti trattati riguarda le motivazioni che hanno spinto gli alunni a scegliere il percorso binazionale. I candidati dovevano dare un giudizio, su una scala da per niente d'accordo a molto d'accordo, alle seguenti opzioni: perché è proposto dalla mia scuola; interesse alla lingua e cultura francese; per le origini francesi della mia famiglia; per interessi futuri; per ottenere un doppio diploma; per sviluppare un sentimento di cittadinanza europeo; per integrarmi a livello europeo. Dall'analisi effettuata, risulta che la motivazione principale nella frequentazione dell'Esabac è il voler ottenere un doppio diploma. La seconda scelta che ha ricevuto un giudizio positivo è l'idea che il percorso di studi provveda a delle migliori prospettive future; segue poi l'interesse alla lingua e alla cultura francese. Per quel che riguarda le opzioni valutate più negativamente, i candidati si dimostrano in disaccordo con perché è proposto dalla mia scuola, per integrarmi a livello europeo e per soiluppare un sentimento di cittadinanza europeo (3).

Gli alunni sono in seguito stati pregati di dare un giudizio del livello di lingua francese, rispettivamente, dei loro docenti di lingua e letteratura e di storia. In rapporto al primo gruppo la valutazione risulta positiva: la media aritmetica delle risposte date per ogni istituto si situa infatti tra la risposta abbastanza alto e molto alto. Anche nei confronti degli insegnanti di storia, a partire dalla medesima comparazione delle risposte ottenute per ogni scuola, risulta una tendenza positiva, situata tra abbastanza alto e molto alto.

Ai candidati è stata conseguentemente richiesta un'opinione riguardo la strutturazione del programma delle due discipline specifiche. Servendosi di una scala da totalmente in disaccordo a totalmente d'accordo, dovevano indicare se, secondo loro, le caratteristiche principali del dispositivo descritte nei decreti ministeriali sono effettivamente trasmesse e trattate in classe. Le opzioni proposte sono le seguenti: studio della letteratura italiana; interdisciplinarità in classe; utilizzo chiaro della metodologia; la storia insegna a divenire un cittadino europeo; studio della storia italiana; studio della letteratura francese; utilizzo di un approccio tematico in classe di storia; studio della storia europea; studio della letteratura europea; studio della storia francese; integrazione linguistica a livello europeo; utilizzazione di itinerari letterari; studio della storia mondiale. Secondo l'analisi effettuata, gli elementi del programma trasmessi in maniera più critica (totalmente in disaccordo), sono lo studio della storia italiana e lo studio della letteratura italiana ed europea. A riguardo dell' interdisciplinarità durante i corsi e della trasmissione dei principi di cittadinanza europea in classe di storia, $\mathrm{i}$ candidati si dimostrano invece abbastanza d'accordo. Le restanti opzioni si situano infine sotto la voce abbastanza d'accordo.

In seguito è d'interesse scoprire per quanto tempo i docenti delle discipline specifiche parlino effettivamente in lingua francese in clas- 
«Un esame, due diplomi»: I'ESABAC, una ricerca empirica nella regione Toscana

V. Rocchi, G. SCHLEMmingeR se. Gli alunni dovevano quindi scegliere tra: meno della metà del tempo, la metà del tempo, la maggior parte del tempo, sempre. Il risultato ottenuto nei confronti degli insegnanti di lingua e letteratura a partire dalla media aritmetica dei valori per ogni istituto, coincide con la risposta la maggior parte del tempo. La tendenza è perciò positiva. La stessa domanda è stata posta a riguardo dei docenti di storia: attraverso la medesima comparazione tra le scuole si ottiene una media corrispondente alla risposta la maggior parte del tempo. Come nel primo caso dunque, gli insegnanti della disciplina non linguistica parlano per la maggior parte del tempo in lingua francese durante le loro lezioni.

Un altro punto della ricerca riguarda le attività praticate dagli alunni al di fuori del contesto scolastico per approfondire e migliorare la loro conoscenza della lingua francese. Più in dettaglio, sono stati proposti: scambi e/o contatti con madrelingua; il guardare la tv; l'ascolto di musica; il leggere in lingua e, più in generale, l'approfondimento grammaticale, lessicale ecc. del francese. Le medie indicano che gli allievi praticano sporadicamente queste attività. Quelle meno gettonate sono effettuate come segue: 1) letture e scambi con madrelingua raramente; 2) l'approfondimento della lingua e il guardare la TV meno di una volta al mese. Infine, l'ascolto di musica è il più sovente, con una frequenza di una volta al mese. Conseguentemente il questionario si è concentrato sulla percezione che i candidati hanno del proprio livello in lingua francese. L'obiettivo è quello di ottenere un quadro generale sulla soddisfazione nei confronti del dispositivo, in particolare, sul grado di conoscenza della lingua straniera che esso riesce a veicolare. A partire dai risultati ottenuti, la maggioranza dichiara di essere abbastanza soddisfatta: la valutazione è quindi positiva.

Due ulteriori domande mirano a scoprire quali prospettive future sono prese in considerazione dagli allievi dopo l'ottenimento del diploma italo-francese. Per meglio poter analizzare le informazioni ottenute, le risposte sono state suddivise in due gruppi: il primo riguardante coloro che prevedono di continuare il percorso frequentando un istituto di istruzione superiore, il secondo prendente in considerazione coloro che invece hanno intenzione di entrare nel mondo del lavoro. Nel primo caso, la maggioranza (58\%) ha indicato voler rimanere in Italia. Di questi, il 39\% non vuole continuare a praticare la lingua francese. Dei restanti candidati, il 28\% desidera andare in Francia. Infine il $14 \%$ ha indicato l'opzione altro. Nel caso dei futuri lavoratori, i risultati si presentano più omogenei: il $37 \%$ afferma voler cercare un impiego in Francia, il 39\% in Italia, il 24\% altrove.

L'inchiesta termina con due domande aperte nelle quali agli alunni è domandato, rispettivamente, di indicare a quale istituto di istruzione si sono scritti (primo quesito) o per quale lavoro hanno fatto doman- 
«Un esame, due diplomi»: I'ESABAC, una ricerca empirica nella regione Toscana

V. ROCCH, G. SCHLEMMINGER

\section{CONCLUSIONI}

da (secondo quesito). Nel primo caso hanno dato un feedback 81 persone su 172. Le risposte ottenute sono state suddivise secondo lo stato, Francia o Italia, e secondo le loro città e facoltà. Alla luce di ciò, 46 persone affermano di voler studiare in Italia, i restanti 35 hanno intenzione di recarsi in Francia, in altri Stati oppure non lo sanno esattamente. Le città indicate in Italia sono, per la maggior parte, situate in Toscana, soprattutto Firenze e Pisa. Alternativa è la regione Lombardia, in particolare Milano. Per quel che riguarda le facoltà scelte, la maggioranza (13) non ha dato alcuna risposta. I restanti hanno invece indicato economia ( 6 persone), lingue e letterature straniere ( 5 persone) e medicina ( 4 persone). Nel caso di coloro che invece vogliono iscriversi in Francia, le città più gettonate si trovano in maggioranza nel sud del paese, a Lione, Grenoble o Nizza. Le facoltà scelte sono chimica/biologia, lingue straniere applicate, matematica, scienze della motricità e architettura. Per quel che riguarda la domanda sul posto di lavoro al quale gli alunni si interessano, la maggioranza non ha risposto (116) o ha indicato di non saperlo ancora (42). Tra coloro che hanno rilasciato delle informazioni (20 su 178), 12 persone vogliono restare in Italia. Per il resto 6 alunni hanno indicato altri Stati e 2 la Francia.

Attraverso l'utilizzo di due metodi di ricerca, un'analisi qualitativa, per gli insegnanti, ed un'analisi quantitativa, per gli alunni, la ricerca qui presentata si è prefissata l'obiettivo di dare un quadro generale delle forze e delle debolezze del dispositivo Esabac nella regione Toscana. Dai risultati del sondaggio ai docenti è stato possibile ottenere una panoramica dei loro metodi di insegnamento. Le domande effettuate hanno permesso, in particolare, di porre l'accento sulla trasmissione da loro attuata del programma e della metodologia, nonché di scoprire eventuali problematiche del dispositivo. Nel caso degli alunni, l'obiettivo era invece quello di comprendere meglio le motivazioni della scelta dell'Esabac, le prospettive future e le attività legate alla lingua francese, appoggiandosi nel frattempo su un'analisi statistica delle risposte per controllarne la validità.

Più in particolare, le conclusioni che si possono trarre riguardo il sondaggio agli insegnanti indicano che $\mathrm{i}$ docenti presentano delle difficoltà soprattutto nel trattare il programma in modo completo e dettagliato. Essi riscontrano inoltre delle problematiche nell'introdurre percorsi interdisciplinari in classe. A causa dell' ampio programma da trattare, gli scambi con i colleghi, per legare interdisciplinarmente le tematiche delle rispettive materie, sono infatti di difficile attuazione.

Per quel che riguarda i punti positivi messi evidenza dalla ricerca, vi è una soddisfazione generale verso il dispositivo. Il doppio 
«Un esame, due diplomi»: I'ESABAC, una ricerca empirica nella regione Toscana

V. ROCCH, G. SCHLEMMINGER

\section{RIFERIMENTI}

diploma è apprezzato, in particolare in rapporto alle sua apertura linguistica e storica verso la Francia e l'Europa. Infine, tra i miglioramenti possibili indicati nelle risposte aperte, $\mathrm{i}$ docenti desidererebbero del materiale didattico apposito (in particolare per la materia di storia) nonché un incremento e un approfondimento delle formazioni linguistiche e metodologiche.

I risultati del questionario agli alunni fanno invece affiorare delle critiche particolarmente riguardo la strutturazione del programma Esabac, soprattutto nello studio della letteratura e della storia italiana. Secondo i candidati infatti, queste tematiche non sono abbastanza approfondite. Inoltre, riguardo la scrittura dei testi (analisi e composizioni), la metodologia utilizzata è poco chiara e non abbastanza trattata. D'altro canto, l'interculturalità del percorso è percepita come uno degli aspetti più positivi e più motivanti. Una delle ragioni per la scelta del dispositivo è infatti l'apertura verso uno stile di vita plurilingue e d'integrazione europea. In linea con ciò, gli alunni si dimostrano piuttosto soddisfatti sia con il funzionamento dell'Esabac, sia con il livello in lingua francese che presentano alla fine del percorso scolastico. Per quel che riguarda le prospettive dopo l'ottenimento del doppio diploma, la tendenza generale indica la scelta di rimanere in Italia piuttosto che in Francia. La continuazione degli studi o della ricerca di un lavoro nella nazione transalpina non rientra dunque tra le prime opzioni contemplate.

In conclusione, i risultati, da un lato, confermano la buona trasmissione di alcuni aspetti fondamentali che l'Esabac prevede nei confronti di alunni ed insegnanti; dall'altro, propongono delle idee per un eventuale miglioramento del dispositivo (in particolare nella strutturazione dei programmi delle discipline specifiche e nelle esigenze del corpo docente). Per questi ed altri motivi la ricerca può gettare le basi per ulteriori studi più dettagliati, basati su una più lunga scala temporale.

Accordo intergovernativo 24 febbraio 2009. Accordo fra il governo della repubblica francese e il governo della Repubblica italiana relativo al doppio rilascio del diploma di Baccalauréat e del diploma d'Esame di stato.

Bulletin Officiel Spécial du 17 juin 2010 (arrêté du 2.6.2010). Double délivrance du diplôme du baccalauréat et du diplôme de l'Esame di Stato.

Bulletin Officiel $\mathrm{n}^{\circ} 46$ du 16 décembre 2010 (note de service $\mathrm{n}^{\circ}$ 2010-227 du 30-11-2010). Épreuves d'histoire-géographie et de langue et littérature italiennes dans les sections binationales 
«Un esame, due diplomi»:

I'ESABAC, una ricerca empirica nella regione

Toscana

V. Rocchi, G. SCHLEMmingeR

\section{PAGINE-WEB}

(ULTIMA

CONSULTAZIONE

21.01.2015)
Esabac.

David H. 2012. L'ESABAC, diplôme binational italo-français de fin d'études secondaires: réflexions sur les premiers pas en contexte italien. Éducation et sociétés plurilingues n ${ }^{\circ} 33$ : 53-66.

DM91/2011 "Norme per lo svolgimento degli Esami di Stato nelle sezioni funzionanti presso istituti statali e paritari in cui è attuato il Progetto Esabac (rilascio del doppio diploma italiano e francese)".

DM95/2013 "Norme per lo svolgimento degli Esami di Stato nelle sezioni funzionanti presso istituti statali e paritari in cui è attuato il Progetto- ESABAC (rilascio del doppio diploma italiano e francese)".

Duc V. 2011. ESABAC (diplôme binational franco-italien). Éducation et sociétés plurilingues $\mathrm{n}^{\circ}$ 30: 41-54.

Legge 11 gennaio 2007. Disposizioni in materia di esami di Stato conclusivi dei corsi di studio di istruzione secondaria superiore e delega al Governo in materia di raccordo tra la scuola e le università

Nota MIUROODGOS protocollo 7773. Progetto ESABAC Diploma binazionale italo-francese (Esame di Stato e Baccalauréat). Avvio della procedura di selezione delle scuole secondarie superiori interessate a rilasciare il doppio titolo a partire dall'a.s. 2012/2013.

Nota MIUR nr. 1036/2013. Progetto ESABAC - Diploma binazionale italo-francese (Esame di Stato e Baccalauréat). Avvio della procedura di selezione delle scuole secondarie superiori interessate a rilasciare il doppio titolo a partire dall'a.s. 2015/2016.

Regolamento marzo 2010 recante "Revisione dell'assetto ordinamentale, organizzativo e didattico dei licei ai sensi dell'articolo 64, comma 4, del decreto legge 25 giugno 2008, n. 112, convertito dalla legge 6 agosto 2008, n. 133”.

Ambassade de France en Italie. http://www.ambafrance-it.org/ Clil. Sito ufficiale dell'Istituto Nazionale di Documentazione, Innovazione e Ricerca Educativa (INDIRE). http://www.indire.it/clil/

Institut français Florence. http://institutfrancais-firenze.com/it Ministère de $\mathrm{l}^{\prime}$ éducation nationale, de l'enseignement supérieur et de la recherche.

http://www.education.gouv.fr/

MIUR. http://www.istruzione.it/ 
«Un esame, due diplomi»:

I'ESABAC, una ricerca

empirica nella regione

Toscana

V. ROCch, G. SCHLEMMINGER

PAGINE-WEB

DEGLI ISTITUTI

SCOLASTICI PRESI IN

CONSIDERAZIONE
Liceo E. Fermi Castel del Piano. http://www.isisamiataovest.it

Liceo Machiavelli-Capponi.

http://www.liceomachiavelli-capponi.it/

Liceo Cecioni. http://www.liceocecioni.govit/

Istituto Sacro Guore. http://www.sacrocuore.com/

Liceo Giovanni da San Giovanni.

http://www.liceoeconomicosociale.it/les/istituto-magistrale-statalegiovanni-da-san-giovanni/

IT Cattaneo. http://itcattaneo.it/

\section{NOTE}

(1) Gli alunni e gli insegnanti presi in considerazione da questa ricerca appartengono alle classi quinte di 6 istituti scolastici toscani, ovvero: l'istituto tecnico "Cattaneo" a San Miniato, il liceo internazionale "Machiavelli-Capponi" a Firenze, il liceo scientifico "E. Fermi" a Castel Del Piano ed infine i licei linguistici "Cecioni", "Giovanni da San Giovanni" e "Sacro Cuore" rispettivamente a Livorno, San Miniato e Firenze.

(2) Nel caso dei docenti di lingua e letteratura i manuali più usati sono i seguenti: 5 persone indicano Langin et al., 2012, Entre les lignes, ed. Löscher; 3 persone Bonini et al. (2009), Écritures, ed. Valmartina ed infine 2 candidati utilizzano Bertrand et al. (2011), Méthodes et Techniques, ed. Nathan. Come testi di integrazione sono stati invece nominati: Agostini et al. (2013), Esabac en Poche, ed. Zanichelli (utilizzato da 6 persone), Méthodes et Techniques, ed. Nathan (utilizzato da 2 persone). I colleghi di storia si servono invece soprattutto di Lambin (2004), Histoire Terminale L. ES. S., ed. Hachette (indicato 3 volte). Come integrazione a questa materia i risultati indicano: Agostini et al. (2013), Esabac en Poche, ed. Zanichelli; Lambin (2004), Histoire Terminale L. ES. S., ed. Hachette; Milza (2005), Histoire de l'Italie: des origines à nos jours, ed. Fayard ed infine testi vari tratti da siti internet.

(3) Nell'ambito di questa domanda, è anche contemplata l'opzione per le origini francesi della mia famiglia, alla quale gli alunni hanno risposto con totalmente in disaccordo. Ciò è dovuto al fatto che, come è stato notato al momento dell'analisi statistica dei questionari, il numero effettivo di candidati aventi origini francesi era molto basso. 